\title{
On the accuracy of surface hopping dynamics in condensed phase non-adiabatic problems
}

\author{
Hsing-Ta Chen ${ }^{1}$ and David R. Reichman ${ }^{1}$ \\ Department of Chemistry, Columbia University, New York, New York 10027, \\ U.S.A.
}

We perform extensive benchmark comparisons of surface hopping dynamics with numerically exact calculations for the spin-boson model over a wide range of energetic and coupling parameters as well as temperature. We find that deviations from golden-rule scaling in the Marcus regime are generally small and depend sensitively on the energetic bias between electronic states. Fewest switched surface hopping (FSSH) is found to be surprisingly accurate over a large swath of parameter space. The inclusion of decoherence corrections via the augmented FSSH (A-FSSH) algorithm improves the accuracy of dynamical behavior compared to exact simulations, but the effects are generally not dramatic, at least for the case of an environment modeled with the commonly used Debye spectral density.

\section{INTRODUCTION}

Electronically non-adiabatic transitions lie at the heart of some of the most important dynamical processes in the physical sciences $\frac{1}{1}$ Phenomena ranging from gas phase atomic and molecular collisions ${ }^{2}$ to electron and energy transfer in the condensed phase ${ }^{\frac{3}{3}}$ are often intimately influenced by the coupling between distinct potential energy surfaces that is induced by nuclear motion. Theoretically, the accurate treatment of non-adiabatic dynamics is challenging, in particular in condensed phase applications where the interplay between the large number of nuclear degrees of freedom with multiple coupled electronic states greatly increases the complexity of the problem. Exact results may be obtained for specific idealized models such as spin-boson systems where potential energy surfaces are harmonic and linearly displaced $\underline{\underline{4} \underline{\underline{7}}}$ In more realistic condensed phase situations, exact solutions are currently out of reach, despite much recent progress $\stackrel{8}{\underline{8}}$

Among the myriad approximate methods for treating non-adiabatic dynamics, the surface hopping approach $^{9}-12$ stands out for several reasons. First, the method is equally applicable to gas phase and condensed phase problems, and can be used to treat realistic anharmonic nuclear motion on potential energy surfaces, albeit in a classical manner $\stackrel{13}{-15}$ Surface hopping has the advantage that it is naturally formulated in the adiabatic picture, so that it can conveniently be employed in conjunction with electronic structure calculations. The method is also inexpensive, non-perturbative, and provides a superior description of branching processes and detailed balance when compared to other approaches, such as the Ehrenfest method $\stackrel{16}{=}$ Despite these appealing features, surface hopping naturally suffers from several deficiencies $\stackrel{17-20}{=}$ Clearly the description of nuclear motion as classical renders the approach incapable of capturing low temperature effects such as nuclear tunneling on a single potential surface $\stackrel{21}{2}-23^{-}$More generally, while surface hopping does not employ perturbation theory in any parameter, as with nearly all mixed quantum-classical approaches to non-adiabatic dynamics, it cannot be sys- tematically derived from an exact starting point $\underline{24,25,26}$ This fact makes it difficult to evaluate surface hopping's domain of validity.

One long recognized shortcoming of surface hopping is the fact that, in its standard implementation, the algorithm does not provide decoherence for electronic amplitudes. This knowledge has led to the development of important modifications of surface hopping aimed at more accurately describing decoherence $27-40$ In an important recent series of studies, Landry and Subotnik showed that a striking consequence of the neglect of decoherence in surface hopping is the failure to properly capture the golden-rule scaling of the non-adiabatic transfer rate in the Marcus regime $\stackrel{41,42}{=}$ It should be noted, however, that there has been some debate as to just how pervasive this problem is $\underline{43}-\underline{47}$ One of the goals of the present work is to provide an in depth examination of this issue.

More broadly we aim to compare surface hopping, with and without corrections for decoherence, to exact calculations in a model condensed phase system, namely the

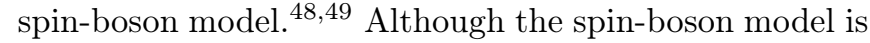
an idealized proxy for a real condensed phase system exhibiting non-adiabatic transitions, it offers the advantage that algorithms now exist that enable the calculation of exact dynamics over a wide swath of the relevant parameter space $\stackrel{\underline{4}}{\underline{\underline{7}}}$ While in the past surface hopping was compared to exact benchmark calculations of low dimensional scattering problems, $\frac{10,11,37}{1}$ we now can provide guidelines for understanding the successes and failures of the surface hopping approach in a broader condensed phase setting. It should be noted, however, that we will restrict our comparison to the "overdamped" case of the coupling to a Debye spectral density, since it is here that facile exact simulations may be performed. While the Debye case represents perhaps the most commonly employed model of a condensed environment in the spin-boson context, our choice implies that some aspects related to the interplay between surface hopping trajectories and decoherence which are expected to be most dramatic and subtle

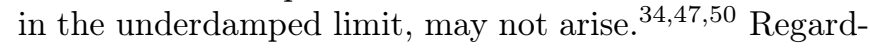
less, our work should at least provide a starting point for assessing how surface hopping performs in generic con- 
densed phase settings.

Our paper is organized as follows: We begin in Sec. II with a review of the standard surface hopping algorithm for the spin-boson model and various formulations of decoherence corrections. In Sec. III, we present our results for the scaling of the non-adiabatic transfer rate with respect to the electronic coupling in the golden-rule regime. In Sec. IV] we explore the full parameter space of spinboson model. We summarize our results and conclude in Sec. V]

\section{FEWEST SWITCH SURFACE HOPPING (FSSH) AND DECOHERENCE}

\section{A. Spin-Boson Model}

We consider the spin-boson model, $H=H_{s}+H_{b}+H_{s b}$, which describes a two-level system with energy bias $\epsilon_{0}$ and constant diabatic coupling $\Delta$

$$
H_{s}=\epsilon_{0} \sigma_{z}+\Delta \sigma_{x}
$$

interacting with an infinite set of harmonic oscillators (bath)

$$
H_{b}=\sum_{j} \frac{1}{2}\left(p_{j}^{2}+\omega_{j}^{2} q_{j}^{2}\right)
$$

where $\omega_{j}$ is the frequency of the $j$-th bath mode $\underline{51}$ The isolated electronic system and the bath are coupled bilinearly

$$
H_{s b}=\sigma_{z} \sum_{j} g_{j} q_{j}
$$

where $g_{j}$ is the coupling strength between the two-level system and the $j$-th harmonic oscillator. We adopt the Pauli matrix notation $\sigma_{x}=|1\rangle\langle 2|+| 2\rangle\langle 1|$ and $\sigma_{z}=$ $|1\rangle\langle 1|-| 2\rangle\langle 2|$ where $|i\rangle$ indicate the diabatic states of the system. Throughout the present paper, we use mass scaled coordinates and momenta for the bath modes, $q_{j}=$ $\sqrt{M_{j}} Q_{j}$ and $p_{j}=P_{j} / \sqrt{M_{j}}$, where $M_{j}$ are the effective mass of nucleus for the $j$-th harmonic oscillator and set $\hbar=1$. We denote bold letters $\boldsymbol{q}, \boldsymbol{p}$ by the vector of nuclear degrees of freedom.

The influence of the bath on the dynamics of the system can be captured in the compact form of a spectral density,

$$
J(\omega)=\frac{\pi}{2} \sum_{j} \frac{g_{j}^{2}}{\omega_{j}} \delta\left(\omega-\omega_{j}\right) .
$$

In the present paper, we consider the Debye model of the spectral density, $\underline{\underline{7}}$

$$
J(\omega)=\frac{E_{r}}{2} \frac{\omega \omega_{c}}{\omega^{2}+\omega_{c}^{2}}
$$

which is appropriate for the description of a solvent environment with Debye dielectric relaxation. The Debye spectral density function is characterized by two parameters, the reorganization energy $E_{r}$, and the characteristic bath frequency $\omega_{c}$. In electron-transfer theory, the reorganization energy represents a direct measure of the coupling strength between the system and the bath. The characteristic frequency is related to the relaxation time scale of the bath, $\tau=1 / \omega_{c}$. The Debye spectral density spans broader frequency than the standard Ohmic $\left(J(\omega) \propto \omega e^{-\omega / \omega_{c}}\right)$ and Brownian forms $\left(J(\omega) \propto \omega /\left(\left(\omega^{2}-\omega_{c}^{2}\right)^{2}+\gamma^{2} \omega^{2}\right)\right)$. Following the procedure outlined in Refs. 52 and 53, it is convenient to discretize the Debye spectral density function via $\omega_{j}=\tan \left((j-0.5) \tan ^{-1}\left(\omega_{\max } / \omega_{c}\right) / N\right)$ where $\omega_{\max }$ is the largest frequency and $N$ is the number of oscillators employed in the discretization.

The population dynamics of the spin-boson model can be calculated by the numerically exact hierarchical equations of motion (HEOM) methodology ${ }_{1}^{4}$ implemented in the Parallel Hierarchy Integrator (PHI) $\stackrel{54}{ \pm}$ The HEOM method is easier to use when the spectral density take the Debye form so that the bath correlation function can be written as a sum of exponentially decaying functions in time $5,55,56$ We use the HEOM method to produce all of our benchmark results for the spin-boson model.

We focus on the reduced population dynamics of the system

$$
P_{i}(t)=\operatorname{Tr}_{b}\left\{\rho(0) e^{i H t}|i\rangle\langle i| e^{-i H t}\right\}
$$

where we assume a factorized initial condition $\rho(0)=$ $\rho_{b}|1\rangle\langle 1|$ and

$$
\rho_{b}=\frac{e^{-\beta H_{b}}}{\operatorname{Tr}_{b}\left\{e^{-\beta H_{b}}\right\}},
$$

with the inverse temperature of the bath, $\beta=1 / k T$. The initial condition of the system corresponds to an impulsive Franck-Condon transition with the bath in a state independent of the system with oscillators centered at $q_{j}=0$.

\section{B. FSSH and its variants}

The fewest-switches surface hopping (FSSH) algorithm is a mixed quantum-classical method that treats the bath degrees of freedom classically and the electronic system quantum mechanically ${ }^{9}-11$ A swarm of classical nuclear trajectories evolve on the adiabatic potential energy surfaces associated with the electronic states with each individual trajectory evolving on a single active surface. Along each trajectory, the electronic wave function propagates according to the Schrodinger equation with the classical nuclear variables evolving as parameters. The essence of FSSH is to simulate the population of the electronic states via the density of trajectories on each 
surface. For this purpose, a surface-hopping scheme is introduced to allow trajectories to hop among the adiabatic energy surfaces and match the electronic populations. The hopping probability of the classical bath trajectories depends on the electronic wave functions with specific conditions for the acceptance of non-adiabatic transitions. Instead of listing these conditions, we describe them within the context of the spin-boson model.

To implement the FSSH algorithm for the spin-boson model, we transform the model to its adiabatic representation by diagonalizing the Hamiltonian $H\left|\Phi_{i}(\boldsymbol{q})\right\rangle=$ $\left(\frac{p^{2}}{2}+V_{i}(\boldsymbol{q})\right)\left|\Phi_{i}(\boldsymbol{q})\right\rangle$ where

$$
V_{i}(\boldsymbol{q})=\frac{1}{2} \sum_{j} \omega_{j}^{2} q_{j}^{2}+(-1)^{i} \sqrt{\left(\boldsymbol{g} \cdot \boldsymbol{q}+\epsilon_{0}\right)^{2}+\Delta^{2}}
$$

are the adiabatic potential energy surfaces and $\boldsymbol{g} \cdot \boldsymbol{q}=$ $\sum_{j} g_{j} q_{j}$. One may transform the diabatic states to the adiabatic representation via the unitary transformation $\left|\Phi_{i}(\boldsymbol{q})\right\rangle=\sum_{j} U_{i j}(\boldsymbol{q})|j\rangle$ where

$$
U(\boldsymbol{q})=\left(\begin{array}{cc}
\sin \theta(\boldsymbol{q}) & -\cos \theta(\boldsymbol{q}) \\
\cos \theta(\boldsymbol{q}) & \sin \theta(\boldsymbol{q})
\end{array}\right)
$$

The adiabatic-diabatic mixing angle is defined as $\theta(\boldsymbol{q})=$ $\frac{1}{2} \tan ^{-1}\left(\Delta /\left(\boldsymbol{g} \cdot \boldsymbol{q}+\epsilon_{0}\right)\right)$ which depends on the bath coordinates. Within the adiabatic representation, the electronic wavefunction can be written as $|\Psi(t)\rangle=$ $c_{1}(t)\left|\Phi_{1}(\boldsymbol{q})\right\rangle+c_{2}(t)\left|\Phi_{2}(\boldsymbol{q})\right\rangle$ and the adiabatic amplitudes satisfy an implicit time-dependent Schrodinger equation

$$
\frac{d}{d t} c_{i}(t)=-i V_{i}(\boldsymbol{q}) c_{i}(t)-\sum_{k} \boldsymbol{p} \cdot \boldsymbol{d}_{i k}(\boldsymbol{q}) c_{k}(t),
$$

where $d_{i k}^{j} \equiv\left\langle\Phi_{i}(\boldsymbol{q})\left|\frac{d}{d q_{j}}\right| \Phi_{k}(\boldsymbol{q})\right\rangle$ is the derivative coupling matrix. For the spin-boson model, the derivative coupling matrix elements are $d_{11}^{j}=d_{22}^{j}=0$ and

$$
d_{12}^{j}=-d_{21}^{j}=\frac{g_{j}}{2} \frac{\Delta}{\left(\boldsymbol{g} \cdot \boldsymbol{q}+\epsilon_{0}\right)^{2}+\Delta^{2}} .
$$

We define the pure state electronic density matrix $\hat{\sigma}$ by $\sigma_{i k}=c_{i} c_{k}^{*}$ and the equivalent equation for the density matrix can be written as

$$
\frac{d}{d t} \hat{\sigma}(t)=-i[\hat{V}(\boldsymbol{q}), \hat{\sigma}(t)]-[\boldsymbol{p} \cdot \hat{\boldsymbol{d}}(\boldsymbol{q}), \hat{\sigma}(t)]
$$

where the potential energy matrix is $V_{i k}(\boldsymbol{q})=\delta_{i k} V_{i}(\boldsymbol{q})$.

The bath in the FSSH algorithm is described via a swarm of trajectories evolving classically on adiabatic potential surfaces. Each individual trajectory propagates on the active adiabatic potential surface, $V_{a}(\boldsymbol{q})$, via $\dot{\boldsymbol{q}}=\boldsymbol{p}$ and $\dot{\boldsymbol{p}}=-\partial V_{a} / \partial \boldsymbol{q}$, and the bath configuration is followed by monitoring the time-dependence of $\left(\boldsymbol{q}^{(n)}, \boldsymbol{p}^{(n)}, a^{(n)}\right)$ for $n=1, \cdots, N_{\text {traj. }}$ Each trajectory is allowed to switch active surfaces in order to force the relative number of trajectories on each surface to mimic the adiabatic probability calculated by the adiabatic amplitudes. To accomplish this, a minimal switching probability for a hop from surface $a$ (active) to surface $b$ (other) during each time step $d t$ may be employed as ${ }^{10}$

$$
\gamma_{a b}^{\text {hop }}=d t \frac{2}{\left|c_{a}\right|^{2}}\left[\operatorname{Im}\left(V_{b a}(\boldsymbol{q}) c_{a} c_{b}^{*}\right)+\operatorname{Re}\left(\boldsymbol{p} \cdot \boldsymbol{d}_{a b} c_{a} c_{b}^{*}\right)\right] .
$$

For the spin-boson model, the hopping probability is determined entirely by the derivative coupling and the adiabatic coherence $c_{a} c_{b}^{*}$. In addition to the hopping probability, trajectories must have enough energy to hop to a new surface and obey energy conservation. If the trajectory switches to a new active surface, the momentum is rescaled in the direction of the derivative coupling by $\boldsymbol{p}^{\prime}=\boldsymbol{p}+\kappa \boldsymbol{d}_{a b}$ satisfying $\left|\boldsymbol{p}+\kappa \boldsymbol{d}_{a b}\right|^{2}+2 V_{b}(\boldsymbol{q})=$ $|\boldsymbol{p}|^{2}+2 V_{a}(\boldsymbol{q})$.

At time $t=0$, we require that the initial configuration of the bath mimics the initial electronic density in the adiabatic representation. The initial configuration for the bath modes are sampled from the thermal Wigner distribution, $\rho_{b} \propto \exp \left\{-\sum_{j} \frac{2}{\omega_{j}} \tanh \left(\frac{\beta \omega_{j}}{2}\right)\left(\frac{1}{2} p_{0 j}^{2}+\right.\right.$ $\left.\left.\frac{1}{2} \omega_{j}^{2} q_{0 j}^{2}\right)\right\}$, with the trace over the bath approximated as $\operatorname{Tr}_{b}\left\{\rho_{b} \cdots\right\} \approx \frac{1}{N_{\text {traj }}} \sum_{\left(\boldsymbol{q}_{0}, \boldsymbol{p}_{0}\right)}^{w} \cdots \equiv\langle\cdots\rangle$. In addition, we initialize the active configuration $a^{(n)}$ accordingly by distributing the initial phase terms on surface 1 with the probability $\left|c_{1}(0)\right|^{2}$ and on surface 2 via probability $\left|c_{2}(0)\right|^{2}$.

Given that the electronic amplitudes are propagated in the adiabatic representation and the bath trajectories move along adiabatic energy surfaces according to the FSSH algorithm, it is non-trivial to extract diabatic electronic populations. We adopt the interpretation of mixed quantum-classical density matrix ${ }^{57}$ for the diabatic population on state $i$, which is given by

$$
\begin{aligned}
P_{i}= & \left\langle\sum_{j}\left|U_{i j}(\boldsymbol{q})\right|^{2} \delta_{j a}\right. \\
& \left.+\sum_{j<k} 2 \operatorname{Re}\left[U_{i j}(\boldsymbol{q}) \sigma_{j k} U_{i k}^{*}(\boldsymbol{q})\right]\right\rangle .
\end{aligned}
$$

Note that the expression for $P_{i}$ includes information from the active surface $(a)$ as well as the adiabatic amplitude $\left(\sigma_{j k}\right)$. For the spin-boson model, we can express the reduced population dynamics of state 1 as

$$
\begin{aligned}
P_{1}=\langle & \left.\sin ^{2} \theta(\boldsymbol{q}) \delta_{1 a}+\cos ^{2} \theta(\boldsymbol{q}) \delta_{2 a}\right\rangle \\
& +\left\langle 2 \sin \theta(\boldsymbol{q}) \cos \theta(\boldsymbol{q}) \operatorname{Re}\left[c_{1} c_{2}^{*}\right]\right\rangle
\end{aligned}
$$

which is composed of a portion associated with the active surface and a portion contributed by the adiabatic coherence.

\section{Decoherence}

Within the standard FSSH algorithm, a difficulty arises when a trajectory passes through the coupling re- 
gion and the electronic wavefunction may bifurcate on

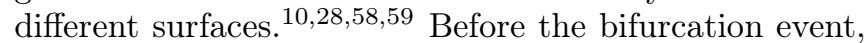
each FSSH trajectory carries a particular electronic amplitude. After the trajectory passes through the coupling region, the wavefunction retains its phase and the density matrix remains pure, even if the trajectories are separated on different surfaces. This failure to incorporate decoherence may lead to an inaccurate description of electronic dynamics.

The augmented FSSH (A-FSSH) $\underline{60}$ has been proposed to resolve this problem by collapsing the electronic state on the inactive surfaces and projecting onto the active surface according to a decoherence rate calculated on the fly. The full procedure of the A-FSSH algorithm is outlined in Ref. 60. Here, for completeness, we briefly review the A-FSSH scheme.

The decoherence rate depends on the matrix of augmented moments of the bath coordinate and momentum $(\delta \hat{\boldsymbol{q}}, \delta \hat{\boldsymbol{p}})$ which provide information regarding the separation of a proxy wave packet in phase space. The augmented moments evolve along a trajectory which follows the equations of motion

$$
\begin{gathered}
\frac{d}{d t} \delta \hat{q}_{j}=\hat{T}_{j}^{q}-T_{j, a a}^{q} \hat{I}, \\
\frac{d}{d t} \delta \hat{p}_{j}=\hat{T}_{j}^{p}-T_{j, a a}^{p} \hat{I},
\end{gathered}
$$

where $\hat{T}^{q}$ and $\hat{T}^{p}$ are obtained by expanding the full quantum Liouville equation to first order in $\hbar$ (linearized approximation)

$$
\begin{gathered}
\hat{T}_{j}^{q} \equiv-i\left[\hat{V}, \delta \hat{q}_{j}\right]+\delta \hat{p}_{j}-\sum_{k} p_{k}\left[\hat{d}_{k}, \delta \hat{q}_{j}\right], \\
\hat{T}_{j}^{p} \equiv-i\left[\hat{V}, \delta \hat{p}_{j}\right]+\frac{1}{2}\left\{\delta \hat{F}_{j}, \hat{\sigma}\right\}-\sum_{k} \hat{p}_{k}\left[\hat{d}_{k}, \delta \hat{p}_{j}\right],
\end{gathered}
$$

and the matrix of forces is given by $\hat{F}_{j} \equiv-\partial \hat{V} /\left.\partial q_{j}\right|_{\boldsymbol{q}}$ and $\delta \hat{F}_{j}=\hat{F}_{j}-F_{j, a a} \hat{I}$. Via the augmented moments, one can derive the off-diagonal correction to the equation of motion for the reduced electronic density matrix,

$$
\frac{d}{d t} \hat{\sigma}=-i[\hat{V}, \hat{\sigma}]-[\boldsymbol{p} \cdot \hat{\boldsymbol{d}}, \hat{\sigma}]+i[\hat{\boldsymbol{F}}, \delta \hat{\boldsymbol{q}}],
$$

which incorporates the decoherence mechanism in the last term. The estimated decoherence rate for the separation of wavepackets on the active surface $a$ and the inactive surface $b$ is of the form

$$
\gamma_{b a}^{\mathrm{d}}=d t\left\{\frac{\left(\boldsymbol{F}_{b b}-\boldsymbol{F}_{a a}\right) \cdot \delta \boldsymbol{q}_{b b}}{2}-2\left|\boldsymbol{F}_{a b} \cdot \delta \boldsymbol{q}_{b b}\right|\right\},
$$

which is obtained by assuming frozen Gaussian wave packets for the bath wavefunction outside of the derivative coupling region $(\hat{\boldsymbol{d}}=0)$ and reducing the decoherence rate for non-zero derivative couplings. The A-FSSH algorithm also permits resetting the augmented moments to avoid the failure of the linearized approximation. The proposed reset rate is given by bifurcate

$$
\gamma_{b a}^{\mathrm{r}}=-d t \frac{\left(\boldsymbol{F}_{b b}-\boldsymbol{F}_{a a}\right) \cdot \delta \boldsymbol{q}_{b b}}{2} .
$$

Note that $\gamma_{b a}^{\mathrm{r}}$ is the negative collapsing rate since the moments become invalid when wavepackets aggregate.

A more traditional approach to decoherence corrections within surface hopping consists of damping the coherence of the density matrix via a pure-dephasinglike rate ${ }^{33,34,61}$ Within this simpler density-matrix approach, we treat the evolution of the adiabatic coherence outside the derivative coupling region $(\hat{\boldsymbol{d}}=0)$ as pure dephasing in a stochastic formulation $\underline{62}$ Inside the zero derivative coupling region, the population transfer is excluded and the adiabatic coherences satisfy $\frac{d}{d t} \sigma_{j k}=-i\left(V_{j}-V_{k}\right) \sigma_{j k}$ and the formal solution is $\sigma_{j k}(t+\tau)=\sigma_{j k}(t)\left\langle\exp \left\{-i \int_{t}^{t+\tau} d t^{\prime}\left[V_{j}\left(t^{\prime}\right)-V_{k}\left(t^{\prime}\right)\right]\right\}\right\rangle_{w}$. The pure-dephasing time within this stochastic formulation is obtained via the energy difference correlation function ${ }^{62}$

$$
\frac{1}{T_{2}^{*}}=\frac{1}{2} \int_{0}^{\infty}\left\langle\left[V_{j}\left(t^{\prime}\right)-V_{k}\left(t^{\prime}\right)\right]\left[V_{j}(0)-V_{k}(0)\right]\right\rangle d t^{\prime} .
$$

To simulate the decay of the adiabatic coherence within the FSSH algorithm, we introduce a decoherence terms that leads to an exponential decay of the adiabatic coherences. In particular, decoherence is modeled as a Poisson process with the probability that a coherence decay occurs in the time interval $[t, t+d t]$ gives by $\operatorname{Prob}\left\{N\left[\sigma_{j k}(t)\right]-N\left[\sigma_{j k}(t+d t)\right]=1\right\}=e^{-d t / T_{2}^{*}} d t / T_{2}^{*} \approx$ $d t / T_{2}^{*}$ where $N\left[\sigma_{j k}(t)\right]$ is the number of trajectories whose density matrix retains coherence. However, for the spin-boson model, estimation of $T_{2}^{*}$ along each trajectory via Eq. (23) is not well defined. To circumvent this problem, we assume the decoherence time scale takes a similar form for each trajectory

$$
\frac{1}{\tau_{j k}(t)}=\frac{1}{2} \int_{0}^{t}\left(V_{j}\left(t^{\prime}\right)-V_{k}\left(t^{\prime}\right)\right)\left(V_{j}(0)-V_{k}(0)\right) d t^{\prime}
$$

which gives an estimate of the pure-dephasing time outside of the derivative coupling region. The decoherence rate for the off-diagonal term $\sigma_{j k}$ is then given by

$$
\gamma_{j k}^{\mathrm{d}}(t)=\frac{d t}{\tau_{j k}(t)} .
$$

A decoherence factor for the off-diagonal density matrix elements may be defined as $\sigma_{j k}=\eta_{j k} c_{j} c_{k}^{*}$, so that the hopping rate, namely the analogy of Eq. (13), becomes

$$
\gamma_{a b}^{\text {hop }}=d t \frac{2}{\left|c_{a}\right|^{2}} \operatorname{Re}\left(\boldsymbol{p} \cdot \boldsymbol{d}_{a b} \sigma_{a b}\right) \text {. }
$$

For every time step, we calculate the decoherence timescale $\tau_{j k}(t)$ by accumulating energy difference correlations along the trajectory. If a decoherence event 

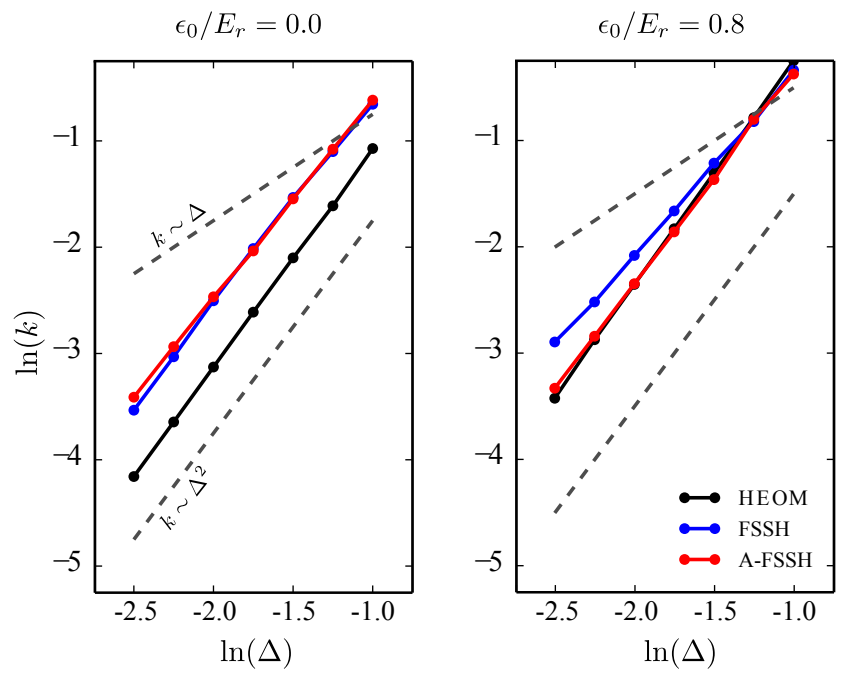

Figure 1. Diabatic population transfer rates $(k)$ as a function of diabatic coupling $\Delta$ for FSSH (blue), A-FSSH (red), and HEOM (black) in the unbiased $\epsilon_{0} / E_{r}=0$ and biased $\epsilon_{0} / E_{r}=0.8$ cases. The bath temperature is assumed to be in the classical limit, $T=300 \mathrm{~K}$. The reorganization energy is $E_{r}=520 \mathrm{~cm}^{-1}$, while the bath frequency scale $\omega_{c}$ is tuned so that $\Delta / \omega_{c} \ll 1$. The dashed lines are reference markers of sub-quadratic and quadratic dependence, respectively. The diabatic population transfer rates is extracted from the population dynamics by exponential fitting.
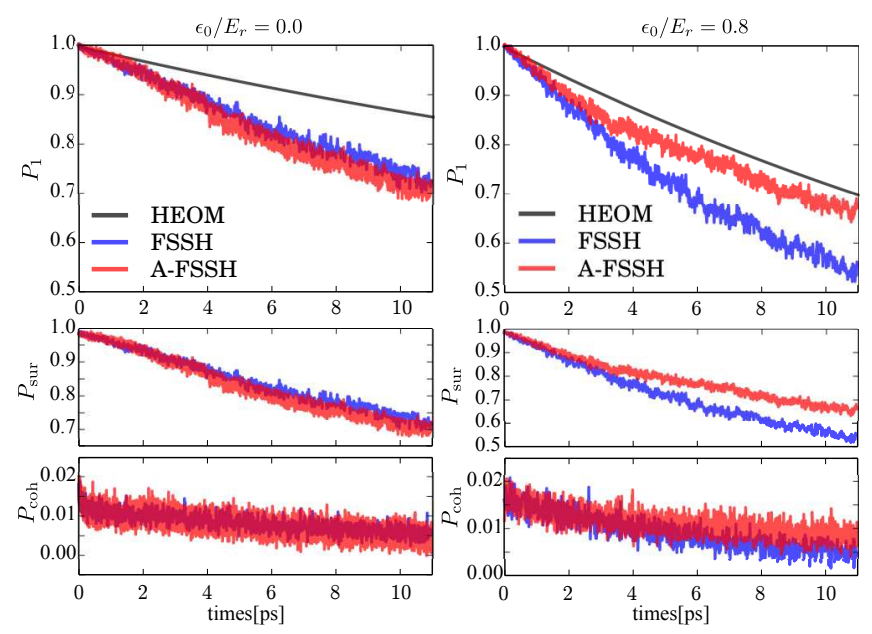

Figure 2. Population dynamics of the for FSSH, A-FSSH, and HEOM in the (a) unbiased $\epsilon_{0} / E_{r}=0$ and (b) biased $\epsilon_{0} / E_{r}=0.8$ cases. The lower panels show the surface and coherence terms separately, as defined in Eq. (27). The bath temperature is $T=300 \mathrm{~K}$, the reorganization energy is $E_{r}=$ $520 \mathrm{~cm}^{-1}$, and $\omega_{c}=85 \mathrm{~cm}^{-1}$.

occurs, the associated factor $\eta_{j k}$ is set to zero. Then we symmetrize the density matrix and continue the trajectory propagation.

\section{THE GOLDEN-RULE REGIME}

A surprising feature of the standard FSSH algorithm that has recently been discovered is its failure to capture the quadratic dependence of the (diabatic) transfer rate in the weak coupling regime. The generality of this behavior remains somewhat controversial. Furthermore, the fundamental origin of this apparent failure is unclear. Landry and Subotnik noted an interesting aspect of the simple one-dimensional Landau-Zener (LZ) problem. ${ }^{41}$ In the standard treatment of the LZ problem with initial electronic population on one surface only, a single voyage through the crossing region produces population differences in harmony with the expected quadratic coupling dependence of the rate. However, if the system is prepared initially with arbitrary population on both diabatic surfaces, then a passage through the crossing point induces a population change that is proportional to both the electronic coupling itself as well as its square. It may then be argued that since traversal of the crossing region mixes the populations, multiple crossings will produce a rate with a sub-quadratic coupling dependence. With the addition of decoherence, however, populations are localized after each crossing, such that the rate always retains its proper quadratic golden-rule form. Below we show that while this argument cannot explain the deviations from Marcus golden-rule behavior exhibited by FSSH, the notion that decoherence can alter the coupling dependence of the rate in a favorable way is indeed correct.

Let us briefly revisit the simple one dimensional LZ example. As in Ref. 41., let us take an electronic propagator of the form $U=\left(\begin{array}{cc}\sqrt{1-\xi} & \sqrt{\xi} \\ -\sqrt{\xi} & \sqrt{1-\xi}\end{array}\right)$, where $\xi=\exp \left[-\frac{2 \pi}{\hbar} \frac{\Delta^{2}}{\mid \boldsymbol{v} \cdot\left(\boldsymbol{F}_{\mathbf{1}}-\boldsymbol{F}_{\mathbf{2}}\right)}\right] \equiv \exp \left[-\eta \Delta^{2}\right]$ is the LZ parameter which depends on the crossing velocity $\boldsymbol{v}$ and the difference in the (diabatic) forces, $\boldsymbol{F}_{\mathbf{1}}-\boldsymbol{F}_{\mathbf{2}}$, at the crossing point, and the electronic coupling, $\Delta$. Clearly a pure initial wave packet with amplitude placed entirely on surface $a$, namely $P(0)=\left(\begin{array}{l}1 \\ 0\end{array}\right)$, produces a population difference on surface $b$ after one crossing that is proportional to $\Delta^{2}$ for small $\Delta$. On the other hand, if the initial packet has the form $P(0)=\left(\begin{array}{c}\alpha \\ \beta\end{array}\right)$ where $\alpha$ and $\beta$ are arbitrary constants satisfying $\alpha^{2}+\beta^{2}=1$, then after one passage the population difference on the surface $b$ is given by $(\xi-1) \beta^{2}+(1-\xi) \alpha^{2}-2 \sqrt{\xi(1-\xi)} \alpha \beta \approx$ $\left(\alpha^{2}-\beta^{2}\right) \eta \Delta^{2}-\sqrt{\eta} \Delta \alpha \beta$. The linear term in the electronic coupling heralds an apparent subquadratic dependence of the rate on $\Delta$. Importantly, however, it should be noted that the mixing of populations that occurs during passage through the crossing region depends on $\Delta$. In particular, starting from the "pure" initial state $P(0)=\left(\begin{array}{l}1 \\ 0\end{array}\right)$, passage through the crossing region produces populations on each diabatic state that are nonzero, but do depend on $\Delta$ and are thus not arbitrary 
constants. Via consideration of $U^{n}\left(\begin{array}{l}1 \\ 0\end{array}\right)$, it is straightforward to demonstrate that even in the absence of decoherence, multiple crossings do not generate spurious terms in the $b$-state population that are linear in $\Delta$ within this simple model.

To explore the issue of the behavior predicted by surface hopping in the Marcus regime, we turn to direct simulation. In Fig. 1, compare the exact diabatic population transfer rates, numerically extracted from HEOM simulations in the high temperature, weak electronic coupling regime to both the results predicted by FSSH as well as the decoherence based A-FSSH algorithm. In both cases, we use Eq. (15) to extract diabatic quantities. The exact HEOM simulations are not confined to the strict high temperature limit. Thus we expect rates that scale as $\Delta^{2}$, but do not necessarily conform quantitatively to standard Marcus theory. The results are shown for both an unbiased and strongly biased cases of the spin-boson problem. Several important features should be noted. First, in the symmetric situation, the FSSH approach yields the correct scaling of the rate with $\Delta$ and produces results that are essentially indistinguishable from those of A-FSSH. This is true even as the electronic coupling is varied over a wider range, and for all values of the reorganization energy. On the other hand, when there is a sizable energetic bias, the rate indeed violates Marcus scaling and behaves in a manner qualitatively similar to that described in Ref. 60.63 Importantly, however, the magnitude of the deviations we find are significantly smaller than that expected from the calculations of Ref. 60. Remarkably, the inclusion of decoherence corrects this failing, producing results in quantitative correspondence with exact numerics. Thus, violations of the expected golden-rule behavior as well as the impact of decoherence in the weak-coupling regime appear to depend sensitively on the electronic bias.

To gain a deeper understanding of this surprising result, we decompose the non-adiabatic population into terms that have an explicit dependence on the dynamics on a given surface the the coherence between surfaces, respectively. It may be shown that Eq. (15) can be recast as

$$
\begin{aligned}
P_{1}= & P_{\text {sur }}+P_{\text {coh }} \\
= & \left\langle\frac{1}{2}+\frac{1}{2} \frac{\boldsymbol{g} \cdot \boldsymbol{q}+\epsilon_{0}}{\sqrt{\left(\boldsymbol{g} \cdot \boldsymbol{q}+\epsilon_{0}\right)^{2}+\Delta^{2}}}\left(\delta_{2 \lambda}-\delta_{1 \lambda}\right)\right\rangle \\
& +\left\langle\frac{\Delta}{\sqrt{\left(\boldsymbol{g} \cdot \boldsymbol{q}+\epsilon_{0}\right)^{2}+\Delta^{2}}} \operatorname{Re}\left[c_{1} c_{2}^{*}\right]\right\rangle,
\end{aligned}
$$

where we have labeled the two relevant terms in Eq. (27) as the "surface" term, $P_{\text {sur }}$, and the "coherence" term, $P_{\text {coh. }}$. Note that we are using the diabatic interpretation of Ref. 57, so in essence it is the "surface" term that is expected to be most sensitive to decoherence corrections applied in the adiabatic basis, not the "coherence" term. Furthermore, note that it is the surface term that has the stronger explicit dependence on the energetic bias, in harmony with the notion that the distinction between FSSH and its decoherence corrected variants will depend on bias as reflected in the way decoherence alters the behavior of the first term of Eq. (15). In Fig. (2) we show the temporal decay of population in both the unbiased and biased cases, within both FSSH and A-FSSH. We also show separately the surface and coherence terms. For the unbiased case, FSSH and A-FSSH yield essentially identical results, while in the biased case A-FSSH is in near quantitative agreement with the exact result while the standard FSSH result decays too rapidly. The difference between the two results is noticeable only in the surface term, which dominates over the coherence term. Thus, we find that distinction between the unbiased and biased cases reflects the manner in which the bias couples to coherence-sensitive terms as exposed in Eq. (27).

\section{THE FULL PARAMETER SPACE}

In this section, we explore more broadly the comparison of surface hopping to benchmark calculations of dynamics in the spin-boson model. Fig. 2 illustrates that in the golden-rule regime, standard FSSH produces results in qualitative agreement with the exact behavior produced by HEOM calculations. The inclusion of decoherence can lead to improved and even quantitatively accurate results, however the improvement over FSSH will depends sensitively on the parameters of the underlying Hamiltonian, such as the energetic bias. Similar behavior is seen away from the weak coupling limit. For intermediate electronic coupling and high temperature $\left(k T / \omega_{c} \gg 1\right)$, a regime often difficult to treat via approximate perturbative approaches, we find that FSSH is quite accurate, with an accuracy that is not altered by inclusion of decoherence within the A-FSSH approach. On the other hand, direct decoherence damping with a pure-dephasing-type rate generally leads to less accurate results than FSSH in this regime, especially when the reorganization energy is large. These observations are illustrated in Fig. 3. In the adiabatic regime, where the electronic coupling is large, we again find that FSSH is in good agreement with the exact behavior of the simulated non-equilibrium populations at high temperatures, especially for large reorganization energies. Some select examples of this comparison are illustrated in Fig. 4. In situations where the reorganization energy is small and the system has no energetic bias, the upper left panel of Fig. 4(a) and the upper right panel of Fig. 5 illustrate how A-FSSH provides a damping of population oscillations that brings the approximate results into quantitative correspondence with exact simulations. With respect to more phenomenological treatments of decoherence, two new features stand out. First, direct decoherence damping with a pure-dephasing rate generally leads to more accurate results in the strong-coupling regime than it does in situations where the electronic coupling is intermedi- 
(a)

(b)
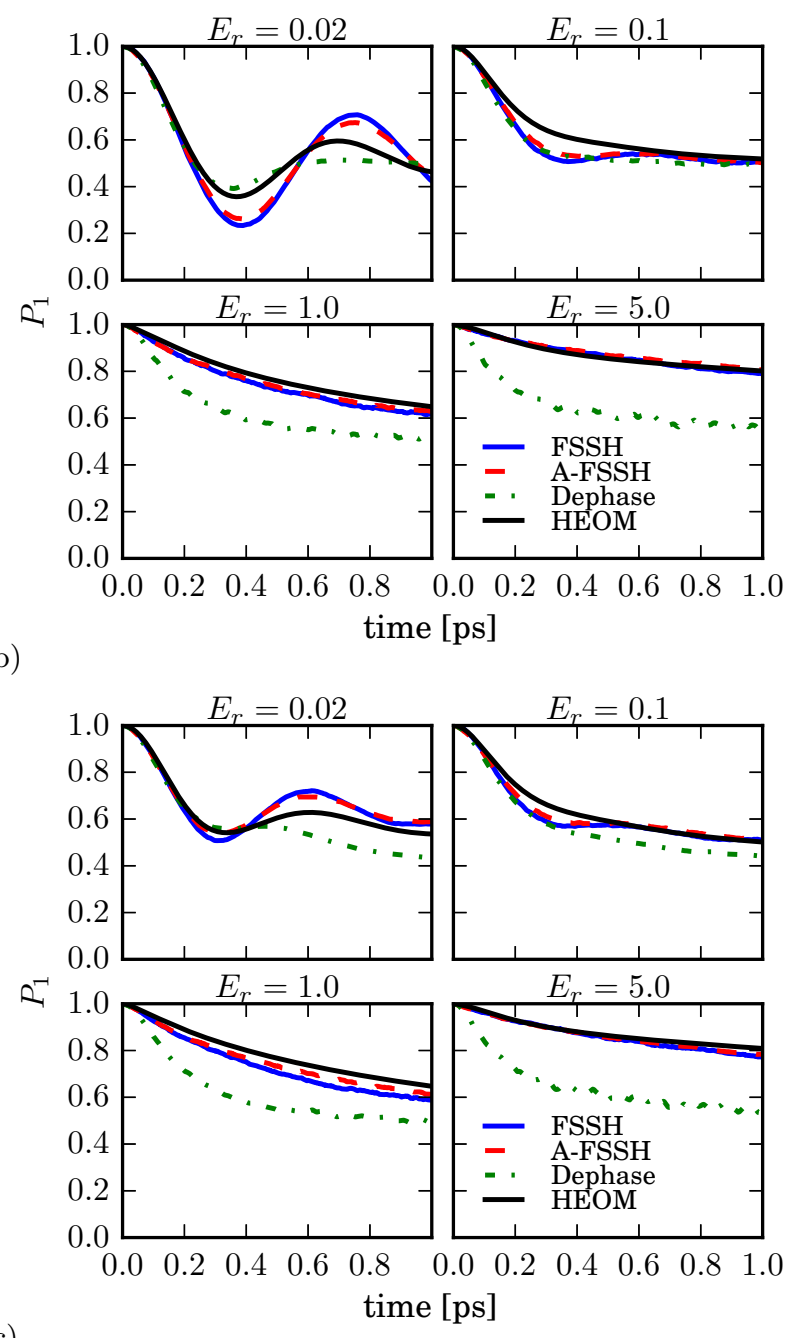

(c)

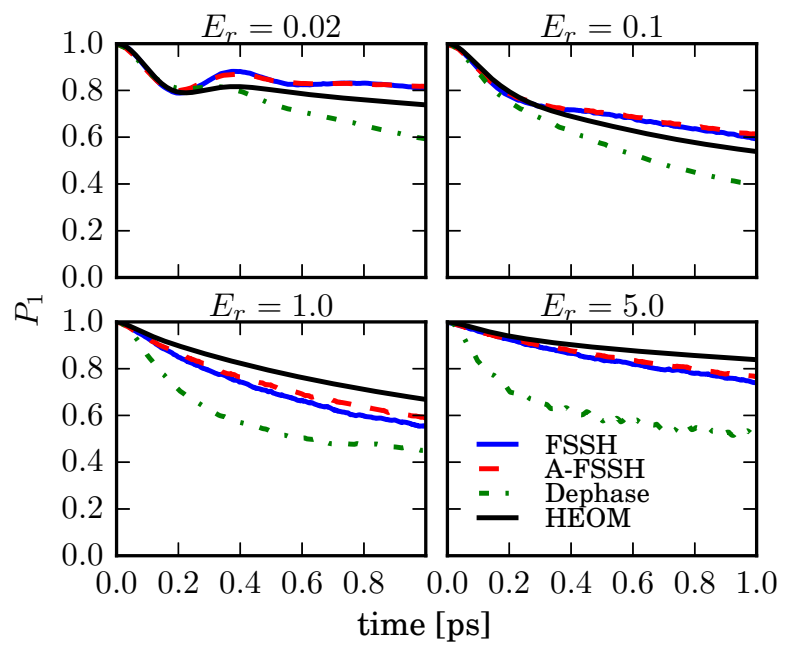

Figure 3. High temperature dynamics with intermediate electronic coupling strength. We employ a reference unit of energy of $104 \mathrm{~cm}^{-1}$. Parameters are $k T=2(T=300 \mathrm{~K})$, $\Delta=\omega_{c}=0.2$, (a) $\epsilon_{0} / \Delta=0$, (b) $\epsilon_{0} / \Delta=2$, and (c) $\epsilon_{0} / \Delta=4$. Reorganization energies are scanned from small to large and are listed on each panel. "Dephase" refers to the use of Eqs. (24)-(26). (a)

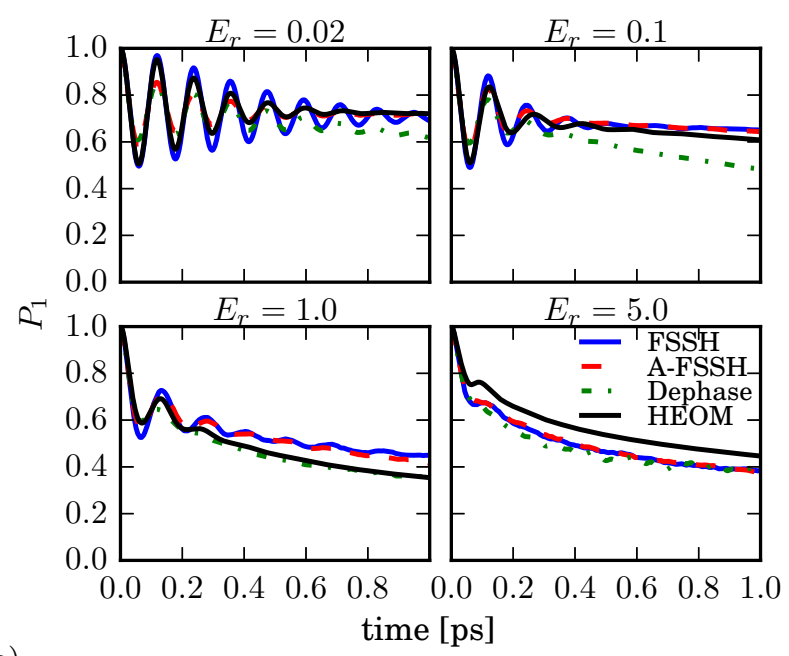

(b)

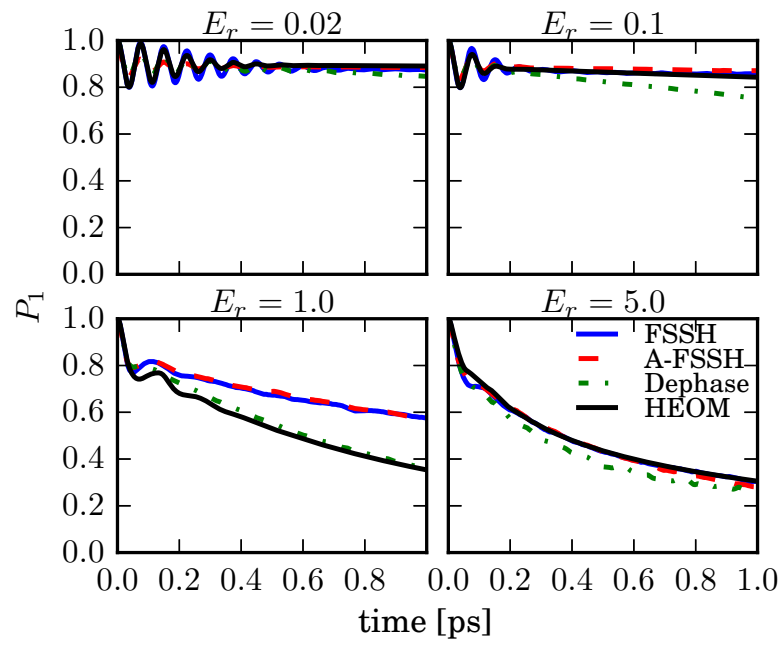

Figure 4. High temperature dynamics in the adiabatic regime. We employ a reference unit of energy of $104 \mathrm{~cm}^{-1}$. Parameters are $k T=2(T=300 \mathrm{~K}), \Delta=1, \omega_{c}=0.2,(\mathrm{a}) \epsilon_{0} / \Delta=2$ and (b) $\epsilon_{0} / \Delta=4$. Reorganization energies are scanned from small to large and are listed on each panel. "Dephase" refers to the use of Eqs. (24)-(26).

ate or small as compared to other energy scales in the problem. In particular, unlike in the case of intermediate coupling, the more phenomenological treatment of decoherence appears not to lead to gross overestimates of the rate of population decay in energetically biased cases for large electronic couplings. Furthermore, we find, for the first time, examples where a simple "pure dephasing" correction leads to clearly improved accuracy over both FSSH and A-FSSH. We emphasize however that in general we find A-FSSH to be, on average, the most accurate approach across the full parameter space.

Lastly, we turn to situations where the temperature is comparable to, or lower than, the characteristic bath frequency. In such situations we expect any surface hopping approach to be unreliable due to the fact that the dynam- 


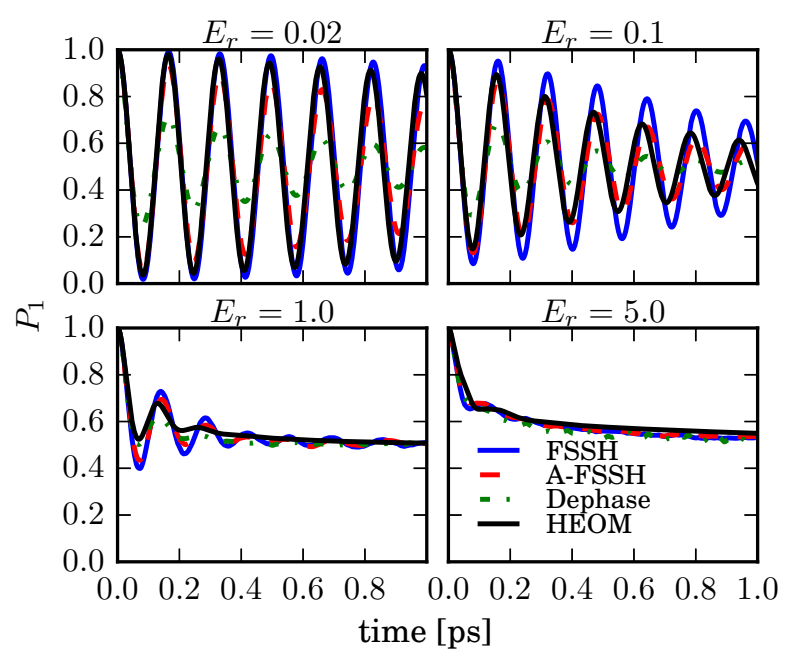

Figure 5. High temperature dynamics in the adiabatic regime. We employ a reference unit of energy of $104 \mathrm{~cm}^{-1}$. Parameters are $k T=2(T=300 \mathrm{~K}), \Delta=1, \omega_{c}=0.2$, and $\epsilon_{0}=0$. Reorganization energies are scanned from small to large and are listed on each panel. "Dephase" refers to the use of Eqs. (24)(26).

ics of the nuclei are treated classically. Thus processes such as nuclear quantum tunneling cannot be described. While we find this to be generally the case, there are situations where the surface hopping approaches find some success even in this "quantum bath" regime. In particular, when the electronic coupling is strong and the time scale is relatively short, both FSSH and A-FSSH can accurately model the Rabi-like oscillations for several periods of motion as illustrated in the upper left panel of Fig. 6 (a) and (b). For intermediate temperatures AFSSH can accurately correct the decay rate of the amplitude of oscillations, however its accuracy diminishes at lower temperatures as shown in Fig. 7. In general, however, surface hopping fails to quantitatively capture population relaxation in these regimes, with some "worstcase" examples illustrated in Fig. 6.

\section{v. CONCLUSIONS}

In this work we have provided, to the best of our knowledge, the first detailed comparison of surface hopping with exact quantum dynamics for an idealized but nontrivial model of condensed phase non-adiabatic dynamics. In particular, we have focused on the role played by decoherence across the entire parameter space in general, and in the incoherent golden-rule regime in particular. Our results provide both an understanding of how decoherence influences behavior in the weak electronic coupling regime as well as general guidelines for the reliability of surface hopping with or without decoherence corrections across all regimes.

With respect to recovery of Marcus golden-rule scaling (a)

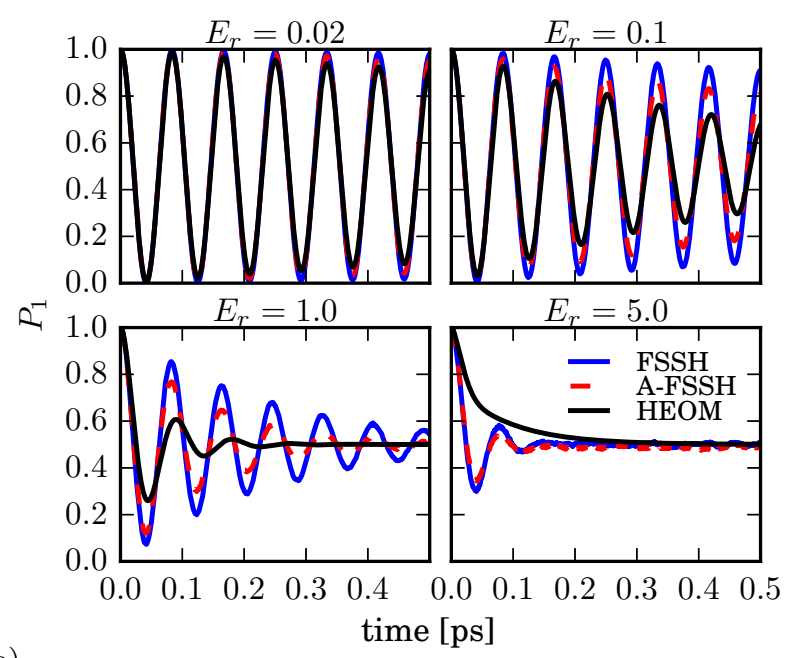

(b)

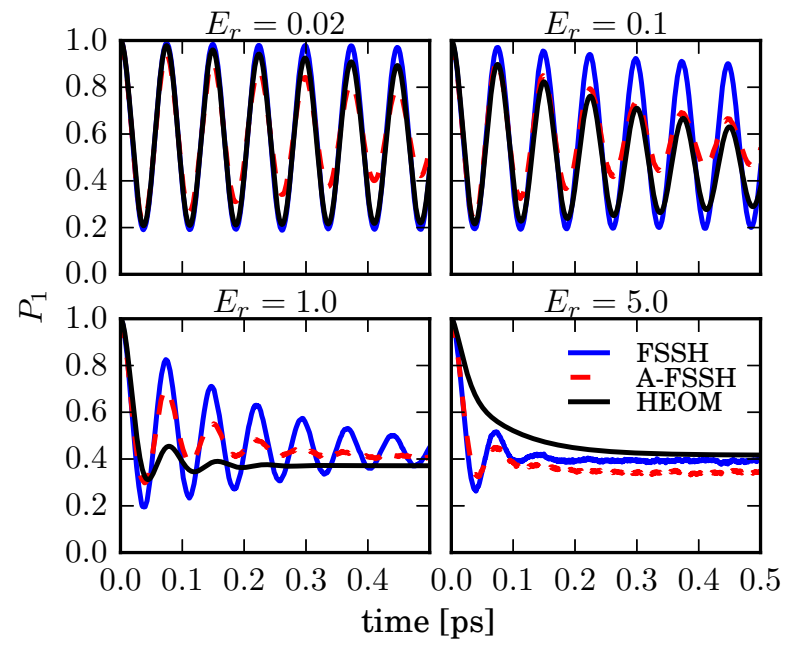

Figure 6. Low temperature dynamics in the intermediate regime. We employ a reference unit of energy of $104 \mathrm{~cm}^{-1}$. Parameters are $k T=0.2(T=30 \mathrm{~K}), \Delta=2, \omega_{c}=2$, $k T=0.2$, (a) $\epsilon_{0} / \Delta=0$, and (b) $\epsilon_{0} / \Delta=2$. Reorganization energies are scanned from small to large and are listed on each panel.

behavior, we present several novel findings. First, we find that deviations from golden-rule scaling, at least within the confines of the spin-boson model with a standard Debye spectral density, do not occur for symmetric systems and only become apparent in systems with a large energetic bias. In biased cases the inclusion of decoherence appears to correct the errant behavior of the standard FSSH approach. On the other hand, we show that the origins of the inability of FSSH to yield golden-rule behavior are subtle and the departure from the quadratic scaling are smaller than expected from past work. Lastly, we note that while it is clear that the decoherence based A-FSSH algorithm alters the electronic dependence of the transfer rate in the weak coupling limit so that the standard golden-rule is recovered, we have no analytical 


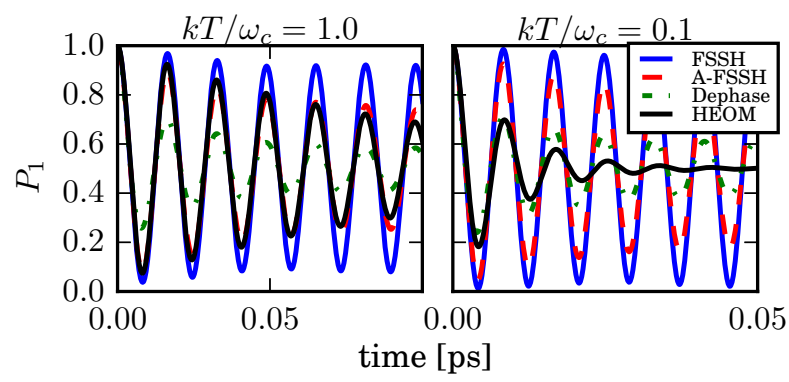

Figure 7. Intermediate and low temperature dynamics in the adiabatic regime. We employ a reference unit of energy of $104 \mathrm{~cm}^{-1} . \epsilon_{0}=0$ and reorganization energy is large $E_{r}=5$. Parameters are (left) $\Delta=10, \omega_{c}=1, k T=1$ and (right) $\Delta=20, \omega_{c}=2, k T=0.2$. "Dephase" refers to the use of Eqs. (24)-(26).

argument that demonstrates that this should occur, or that it will continue to be true over a wider range of $\Delta$ than we have investigated.

A systematic survey of parameter space provides important guidelines concerning the accuracy of surface hopping and its decoherence-corrected variants. One major conclusion that can be immediately reached is that, in general, the standard FSSH is surprisingly accurate in large portions of parameter space. Furthermore, while the decoherence-based A-FSSH approach often leads to some improvement in the description of the temporal decay of non-equilibrium population, on average the corrections are not dramatic. The largest improvements fostered by the inclusion of decoherence provided within the A-FSSH approach are found in the previously discussed golden-rule regime (c.f. Fig. 2) as well as in cases where decoherence damps otherwise oscillatory population decay. Thus, at least with respect condensed phase environments with widely dispersed spectral properties, the standard FSSH approach should generally provide a reasonable description of dynamics.

All of the surface hopping approached we have employed in this work have difficulty in accurately describing low temperature situations, with the exception of symmetric cases where the electronic coupling is so weak that essentially pure Rabi oscillations are observed on short to intermediate time scales. However this breakdown of surface hopping is unsurprising as the approach is incapable of capturing nuclear tunneling effects. Quantitative breakdowns also appear at high and intermediate temperatures not only in the golden-rule limit, but also for intermediate to strong electronic coupling when the coupling to the bath (as given in the reorganization energy) is also sizable. However, even in these regimes failures appear as isolated examples more than generic trends.

We have also investigated decoherence corrections that are perhaps less well justified than that provided by AFSSH but are simpler conceptually. In particular, we have explored an approach similar to the earliest decoherence corrections which employs a simple damping term given by the pure dephasing rate along a trajectory. In general we find that such an approach decoheres relaxation dynamics too strongly, often worsening agreement between the standard FSSH algorithm and the exact results. Somewhat surprisingly however, the degree of decoherence provided by this approach may be seen to quantitatively correct the failures of both FSSH and AFSSH in the "isolated" cases where both fail, namely the regimes of sizable electronic and system-bath couplings mentioned above. This coincidence should be investigated further, as it may foster a deeper understanding of the physics associated with these isolated examples, something that we currently have been unable to provide.

\section{ACKNOWLEDGMENTS}

We would like to thank Joseph Subotnik, Brian Landry, Thomas Markland, Aaron Kelly, and Andrés Montoya-Castillo for extensive discussions. This work was supported by grant NSF CHE-1464802.

\section{REFERENCES}

${ }^{1}$ J. C. Tully, J. Chem. Phys. 137, 22A301 (2012)

${ }^{2} \mathrm{~W}$. H. Miller, Classical limit quantum mechanics and the theory of molecular collisions, Vol. 25 (1974) pp. 69-177.

${ }^{3}$ A. Nitzan, Chemical Dynamics in Condensed Phases: Relaxation, Transfer, c Vol. 8 (2006) pp. 1250-1252.

${ }^{4}$ Y. Tanimura and R. Kubo, J. Phys. Soc. Japan (1989)

${ }^{5}$ D. E. Makarov and N. Makri,

Chem. Phys. Lett. 221, 482 (1994)

${ }^{6}$ N. Makri, J. Math. Phys. 36, 2430 (1995)

${ }^{7} \mathrm{M}$. Thoss, H. Wang, and W. H. Miller,

J. Chem. Phys. 115, 2991 (2001)

${ }^{8}$ M. Ben-Nun, J. Quenneville, and T. J. Martínez,

J. Phys. Chem. A 104, 5161 (2000)

${ }^{9}$ J. C. Tully, J. Chem. Phys. 55, 562 (1971)

${ }^{10}$ J. C. Tully, J. Chem. Phys. 07974, 1061 (1990).

${ }^{11}$ J. C. Tully, Faraday Discuss. 110, 407 (1998)

${ }^{12}$ M. Barbatti, Wiley Interdiscip. Rev. Comput. Mol. Sci. 1, 620 (2011)

${ }^{13} \mathrm{~S}$. Hammes-Schiffer and J. C. Tully,

J. Chem. Phys. 101, 4657 (1994)

${ }^{14}$ S. Hammes-Schiffer and J. C. Tully,

J. Chem. Phys. 103, 8528 (1995)

${ }^{15}$ S. Y. Kim and S. Hammes-Schiffer,

J. Chem. Phys. 124, 244102 (2006)

${ }^{16}$ C.-Y. Hsieh and R. Kapral, Entropy 16, 200 (2013)

${ }^{17}$ U. Müller and G. Stock, J. Chem. Phys. 107, 6230 (1997)

${ }^{18}$ M. S. Topaler, T. C. Allison, D. W. Schwenke, and D. G. Truhlar, J. Phys. Chem. A 102, 1666 (1998)

${ }^{19}$ M. D. Hack, A. M. Wensmann, D. G. Truhlar, M. Ben-Nun, and T. J. Martinez, J. Chem. Phys. 115, 1172 (2001)

${ }^{20} \mathrm{~J}$ E. Subotnik and N. Shenvi,

J. Chem. Phys. 134, 244114 (2011)

${ }^{21}$ M. Ben-Nun and T. J. Martínez, Isr. J. Chem. 47, 75 (2007)

${ }^{22}$ S. R. Billeter, S. P. Webb, T. Iordanov, P. K. Agarwal, and

S. Hammes-Schiffer, J. Chem. Phys. 114, 6925 (2001)

${ }^{23} \mathrm{P}$. K. Agarwal, S. R. Billeter, and S. Hammes-Schiffer, J. Phys. Chem. B 106, 3283 (2002) 
${ }^{24}$ R. Kapral and G. Ciccotti, J. Chem. Phys. 110, 8919 (1999) ${ }^{25}$ J. E. Subotnik, W. Ouyang, and B. R. Landry, J. Chem. Phys. 139, 214107 (2013)

${ }^{26}$ Clearly, Ref. 24 and 25 outline steps towards a complete derivation of the FSSH algorithm starting from the exact equation of motion for the density matrix. In each case, however, there is at least one step that needs to be assumed for which the domain of validity is difficult to assess. It is in this strict sense that we refer to the lack of a systematic derivation of FSSH.

${ }^{27}$ F. Webster, E. T. Wang, P. J. Rossky, and R. A. Friesner, J. Chem. Phys. 100, 4835 (1994)

${ }^{28}$ B. J. Schwartz, E. R. Bittner, O. V. Prezhdo, and P. J. Rossky, J. Chem. Phys. 104, 5942 (1996)

${ }^{29}$ K. F. Wong and P. J. Rossky, J. Chem. Phys. 116, 8418 (2002)

${ }^{30}$ K. F. Wong and P. J. Rossky, J. Chem. Phys. 116, 8429 (2002)

${ }^{31}$ M. J. Bedard-Hearn, R. E. Larsen, and B. J. Schwartz, J. Chem. Phys. 123, 234106 (2005)

${ }^{32}$ R. E. Larsen, M. J. Bedard-Hearn, and B. J. Schwartz, J. Phys. Chem. B 110, 20055 (2006)

${ }^{33}$ C. Zhu, A. W. Jasper, and D. G. Truhlar, J. Chem. Phys. 120, 5543 (2004)

${ }^{34}$ C. Zhu, S. Nangia, A. W. Jasper, and D. G. Truhlar, J. Chem. Phys. 121, 7658 (2004)

${ }^{35} \mathrm{~A}$ W. Jasper and D. G. Truhlar, J. Chem. Phys. 123, 64103 (2005)

${ }^{36}$ I. Horenko, C. Salzmann, B. Schmidt, and C. Schutte, J. Chem. Phys. 117, 11075 (2002)

${ }^{3}$ N. Shenvi, J. E. Subotnik, and W. Yang, J. Chem. Phys. 134, 144102 (2011)

${ }^{38} \mathrm{~J}$. Y. Fang and S. Hammes-Schiffer,

J. Phys. Chem. A 103, 9399 (1999).

${ }^{39}$ J. Y. Fang and S. Hammes-Schiffer, J. Chem. Phys. 110, 11166 (1999)

${ }^{40} \mathrm{O}$. V. Prezhdo and P. J. Rossky, Phys. Rev. Lett. 81, 5294 (1998)

${ }^{41}$ B. R. Landry and J. E. Subotnik, J. Chem. Phys. 135, 1 (2011)
42 J. E. Subotnik, J. Phys. Chem. A 115, 12083 (2011)

${ }^{43}$ R. Jiang and E. L. Sibert, J. Chem. Phys. 136, 224104 (2012)

${ }^{44}$ L. Wang and D. Beljonne, J. Chem. Phys. 139, 064316 (2013)

${ }^{45}$ C. A. Schwerdtfeger, A. V. Soudackov, and S. Hammes-Schiffer, J. Chem. Phys. 140, 034113 (2014)

${ }^{46}$ M. J. Falk, B. R. Landry, and J. E. Subotnik, J. Phys. Chem. B 118, 8108 (2014)

${ }^{47}$ A. Jain and J. E. Subotnik, J. Chem. Phys. 143, 134107 (2015)

${ }^{48}$ A. J. Leggett, S. Chakravarty, A. T. A. Dorsey, M. P. A. Fisher, A. Garg, and W. Zwerger, Rev. Mod. Phys. 59, 1 (1987)

${ }^{49}$ D. Mac Kernan, G. Ciccotti, and R. Kapral, J. Chem. Phys. 116, 2346 (2002)

${ }^{50}$ A. W. Jasper, C. Zhu, S. Nangia, and D. G. Truhlar, Faraday Discuss. 127, 1 (2004)

${ }^{51}$ U. Weiss, Quantum dissipative systems, Vol. 10 (World Scientific Publishing Company Incorporated, 1999).

${ }^{52}$ N. Rekik, C.-Y. Hsieh, H. Freedman, and G. Hanna, J. Chem. Phys. 138, 144106 (2013)

${ }^{53} \mathrm{H}$. Wang, M. Thoss, and W. H. Miller, J. Chem. Phys. 115, 2979 (2001)

${ }^{54}$ J. Strümpfer and K. Schulten, J. Chem. Theory Comput. 8, 2808 (2012)

${ }^{55} \mathrm{~V}$. May and $\mathrm{O}$. Kuhn, Charge and energy transfer dynamics in molecular systems (John Wiley \& Sons, Inc., 2008).

${ }^{56}$ Q. Shi and E. Geva, J. Chem. Phys. 119, 12063 (2003)

${ }^{57}$ B. R. Landry, M. J. Falk, and J. E. Subotnik, J. Chem. Phys. 139, 211101 (2013)

${ }^{58}$ E. R. Bittner and P. J. Rossky, J. Chem. Phys. 103, 8130 (1995)

${ }^{59}$ O. Prezhdo and P. Rossky, J. Chem. Phys. 107, 5863 (1997)

${ }^{60}$ B. R. Landry and J. E. Subotnik, J. Chem. Phys. 137, 0 (2012)

${ }^{61}$ O. Prezhdo and P. Rossky, Phys. Rev. Lett. 81, 5294 (1998)

62 J. Skinner and D. Hsu, J. Phys. Chem. 47, 4931 (1986).

${ }^{63}$ The recently published paper, Jain and Subotnik, J. Phys. Chem. Lett. 6 , 4809 (2015), makes a nearly identical observation. We thank Joseph Subotnik for making us aware of this during the writing of this manuscript. 